BMJ Open

Sport \&

Exercise

Medicine

\title{
Promoting exercise in patients with cancers of the head and neck during COVID-19 and beyond
}

\author{
Kunal K Sindhu (D), Anthony D Nehlsen, Richard L Bakst
}

To cite: Sindhu KK, Nehlsen AD, Bakst RL. Promoting exercise in patients with cancers of the head and neck during COVID-19 and beyond. BMJ Open Sport \& Exercise Medicine 2021;7:e001024. doi:10.1136/ bmjsem-2020-001024

KKS and ADN are joint first authors.
Check for updates

\section{(c) Author(s) (or their} employer(s)) 2021. Re-use permitted under CC BY-NC. No commercial re-use. See rights and permissions. Published by BMJ

Radiation Oncology, Icahn School of Medicine at Mount Sinai, New York, New York, USA

Correspondence to Dr Kunal K Sindhu; kunal.sindhu@mountsinai.org

\section{ABSTRACT}

In recent years, the benefits of exercise in patients with cancer have increasingly been recognised. Unfortunately, patients with, and survivors of, cancers of the head and neck often face a constellation of symptomatic and nutritional obstacles that can interfere with their ability to adhere to structured exercise regimens. The spread of COVID-19 has only added to these challenges. In this Viewpoint, the authors detail the impediments faced by patients with cancers of the head and neck in obtaining sufficient exercise, review the evidence supporting the benefits of exercise in patients with cancer and discuss interventions to promote exercise in this patient population.

As patients with cancers of the head and neck experience longer life expectancies after oncologic treatment, medical care teams must devote more time and resources to educating patients about the importance of exercise and assisting them in designing individualised exercise routines.

In recent years, the benefits of exercise in patients with cancer have increasingly been recognised. Unfortunately, patients with, and survivors of, cancers of the head and neck often face a constellation of symptomatic and nutritional obstacles that can interfere with their ability to adhere to structured exercise regimens. The spread of COVID-19 has only added to these challenges. In this Viewpoint, we will detail the impediments faced by patients with cancers of the head and neck in obtaining sufficient exercise, review the evidence supporting the benefits of exercise in patients with cancer and discuss interventions to promote exercise in this patient population.

Patients with cancers of the head and neck face unique challenges in adhering to exercise routines. Not only are nearly a quarter above the age of 70 at the time of diagnosis, but many also report extensive tobacco and alcohol histories and harbour serious comorbidities, including diseases of the heart and lungs. Additionally, patients with cancers of the head and neck who undergo definitive

\section{Keypoints}

- In recent years, the benefits of exercise in patients with cancer have increasingly been recognised.

- Patients with cancers of the head and neck face unique challenges in adhering to exercise routines.

- The COVID-19 pandemic has further complicated the task of adhering to structured exercise regimens for patients with and survivors of head and neck cancer

chemoradiotherapy may experience significant unintentional weight and muscle loss prior to and during treatment, which may compromise subsequent quality of life. Even after treatment, head and neck cancer survivors report several persistent symptomatic barriers to exercise, some of which may be related to oncologic treatment. In a 2018 survey of 437 head and neck cancer survivors in the UK, for example, the most commonly cited symptomatic barriers to exercise included dry mouth or throat $(40 \%)$, fatigue $(37 \%)$, shortness of breath $(30 \%)$, muscle weakness $(28 \%)$ and difficulty swallowing $(25 \%){ }^{1}$

As a result, patients with cancers of the head and neck frequently struggle to obtain sufficient exercise, both during and after oncologic treatment. While data are limited, a 2006 survey among head and neck cancer survivors at an academic oncology clinic found that the total amount of time they spent exercising dropped $70 \%$ after diagnosis. Whereas $30.5 \%$ of patients met public health guidelines for exercise prior to diagnosis, just $8.5 \%$ did so after diagnosis. ${ }^{2}$

This is unfortunate, as the evidence supporting the importance of exercise in improving outcomes in patients with cancer continues to grow. A recent systematic review found that physical activity before, during and after completing oncologic treatment was associated with improvements in cancer-related fatigue, physical function and quality-of-life measures in most of the studies examined. ${ }^{3}$ Another large review of 
136 studies showed that patients with the highest levels of physical activity pre-diagnosis and post-diagnosis had improved overall survival. ${ }^{4}$ This growing body of literature has led to the development of guidelines, like those published by the American College of Sports Medicine, that detail strategies for pre-exercise fitness testing and cancer-specific exercise programmes for patients with breast, colon, gynaecologic, haematologic and prostate cancers. ${ }^{5}$

Regrettably, patients with cancers of the head and neck have been under-represented in studies investigating the impact of exercise on cancer outcomes and quality of life. As a result, guidelines specifically tailored to these patients are lacking. One review published in 2015, for example, found just 16 publications examining the impact of exercise in patients with cancers of the head and neck. While the data were largely heterogeneous, it suggested that physical activity was safe, feasible and associated with improvements in coping with treatment-related side effects. ${ }^{6}$ While these data are encouraging, significant questions remain regarding how to best incorporate exercise into existing treatment paradigms. Further investigations to evaluate the timing, intensity, quantity and types of exercise needed to achieve optimal results are needed.

Historically, physicians treating patients with head and neck cancer have focused primarily on getting patients through short-term oncologic treatments (surgery, chemotherapy and radiotherapy). However, as local and systemic therapies improve and patients with cancers of the head and neck experience longer life expectancies after completing oncologic treatment, it is essential that all physicians who care for these patients begin to place greater emphasis on interventions, such as exercise, that improve quality of life and reduce the risk of death from competing causes. Formally educating patients about the benefits of exercise and involving them in shared decision-making shortly after diagnosis, and before the start of treatment, may help reinforce the long-term importance of maintaining an active lifestyle. To improve compliance, physicians should actively involve family members and personalise exercise goals to fit the needs of each individual patient. ${ }^{7}$ Additionally, in order to overcome the many long-term challenges inherent in maintaining structured exercise routines in this patient population, more resources must be allocated to cancer survivorship initiatives. A multidisciplinary approach that includes physicians, nutritionists, social workers and other members of the medical care team and extends long after the completion of treatment is necessary to overcome the complex symptomatic and nutritional barriers to exercise for long-term survivors of head and neck cancer.

The COVID-19 pandemic has further complicated the task of adhering to structured exercise regimens for patients with and survivors of head and neck cancer, many of whom, by the nature of their age and comorbidities, are at elevated risk of developing severe complications from the SARS-CoV-2. Persistent health concerns about SARS-CoV-2, social distancing requirements enacted by governments seeking to control the virus and the widespread closure of gyms and exercise studios have impacted the ability of patients to maintain structured exercise routines. In response, physicians must continue to encourage and work with their patients to develop individualised, sustainable exercise regimens that may be completed at home. Exercising at home has been shown to be associated with improved long-term exercise compliance and provides a safe and socially distanced way to remain active during the pandemic. ${ }^{7}$ Patients may, for example, take brisk walks, participate in virtual exercise classes and/or (if they have access to sufficient financial resources) invest in home exercise equipment. Given the persistence of the pandemic, it is crucial that our patients continue to obtain sufficient exercise in order to optimise their long-term health.

Exercise is a key component of maintaining a healthy lifestyle. While more research is needed, especially in the context of patients with cancers of the head and neck, the substantial benefits of exercise in regards to improvements in quality of life and overall survival in patients with cancer are increasingly being recognised. As patients with cancers of the head and neck experience longer life expectancies after oncologic treatment, medical care teams must devote more time and resources to educating patients about the importance of exercise and assisting them in designing individualised exercise routines. This is especially true now, as the societal and health restrictions resulting from the spread of the COVID-19 pandemic have made it more difficult than ever for patients to obtain sufficient exercise.

\section{Twitter Kunal K Sindhu @sindhu_kunal}

Contributors KKS and ADN drafted the manuscript. RLB assisted in drafting and revising the manuscript. All authors contributed in conceiving the manuscript.

Funding The authors have not declared a specific grant for this research from any funding agency in the public, commercial or not-for-profit sectors.

Competing interests None declared.

Patient consent for publication Not required.

Provenance and peer review Not commissioned; externally peer reviewed.

Open access This is an open access article distributed in accordance with the Creative Commons Attribution Non Commercial (CC BY-NC 4.0) license, which permits others to distribute, remix, adapt, build upon this work noncommercially, and license their derivative works on different terms, provided the original work is properly cited, appropriate credit is given, any changes made indicated, and the use is non-commercial. See: http://creativecommons.org/ licenses/by-nc/4.0/.

\section{ORCID iD}

Kunal K Sindhu http://orcid.org/0000-0001-9376-8307

\section{REFERENCES}

1 Midgley AW, Lowe D, Levy AR, et al. Exercise program design considerations for head and neck cancer survivors. Eur Arch Otorhinolaryngol 2018;275:169-79.

2 Rogers LQ, Courneya KS, Robbins KT, et al. Physical activity and quality of life in head and neck cancer survivors. Support Care Cancer 2006;14:1012-9.

3 Stout NL, Baima J, Swisher AK, et al. A systematic review of exercise systematic reviews in the cancer literature (2005-2017). Pm $R$ 2017;9:S347-84.

4 Friedenreich CM, Stone CR, Cheung WY, et al. Physical activity and mortality in cancer survivors: a systematic review and meta-analysis. JNCI Cancer Spectr 2020;4:pkz080. 
5 Schmitz KH, Courneya KS, Matthews C, et al. American College of sports medicine roundtable on exercise guidelines for cancer survivors. Med Sci Sports Exerc 2010;42:1409-26.

6 Capozzi LC, Nishimura KC, McNeely ML, et al. The impact of physical activity on health-related fitness and quality of life for patients with head and neck cancer: a systematic review. Br J Sports Med 2016;50:325-38

7 Dalle Grave R, Calugi S, Centis E, et al. Cognitive-Behaviora strategies to increase the adherence to exercise in the management of obesity. J Obes 2011;2011:1-11. 